Diánoia, vol. 18, no. 18, 1972

\title{
REFLEXIONES SOBRE DOS PROBLEMAS DE LA LÓGICA FORMAL
}

\section{Observaciones introductorias}

1. La gran generalización moderna de la lógica formal, que comenzó hace cerca de siglo y medio y ha producido la lógica simbólica, se enfrenta aún a algunos problemas teóricos fundamentales. Desde luego que este hecho no es de por sí sorprendente, pues la lógica simbólica es una disciplina muy joven. Sin embargo, el más serio de sus problemas no resueltos no tiene vigencia únicamente para ella, sino que también la tiene retroactivamente, por así decirlo, para la lógica tradicional aristotélica. Es decir, se plantea para la lógica formal en general. Se trata del problema de la consistencia de algunos de los conceptos fundadores de la lógica, los cuales entran en sus principios básicos. Por ejemplo, está en juego el concepto de clase.

Es como si sólo recientemente se hubiera descubierto y entendido plenamente un problema que siempre estuvo alli, a la vista de todos aquellos a quienes se les hubiera ocurrido buscarlo. Este descubrimiento se hizo en el umbral del presente siglo, justo a tiempo para frustrar cualquier optimismo puro que pudiera haberse engendrado por los grandes avances entonces hechos en lógica simbólica. El momento histórico quedó señalado, en 1901, por la publicación de la brillante paradoja de Russell sobre las clases. ${ }^{1}$

El resultado ha sido que, en nuestro siglo, la lógica simbólica se ha desarrollado en un clima intelectual que está fuertemente teñido de escepticismo acerca de la certeza de los principios intuitivos de la lógica formal. Además, este escepticismo ha contagiado en forma sutil otros aspectos de la teoría lógica. Por ejemplo, la interpretación del sentido lógico de las conectivas proposicionales del cálculo proposicional plantea un problema muy difícil. Los lógicos simbólicos en su mayor parte han aceptado la llamada "interpretación material" de estas conectivas, aunque están muy conscientes de que esta interpretación deja mucho que desear. Existe una disposición a creer que una solución teórica, plenamente satisfactoria, de este y otros problemas es una meta ilusoria. Hay en el aire un pesimismo teórico muy sutil y penetrante.

Ahora bien, no me parece que este pesimismo se justifique. En realidad,

1 Russell comenzó con la consideración de que algunas clases, como la clase de las clases que tienen más de cinco miembros, satisfacen su propia condición de membresía, en tanto que no sucede lo mismo con otras clases, como la clase de los hombres. Podemos, pues, preguntar: ¿es la clase de las clases que no son miembros de sí mismas miembro de sí misma? La respuesta a esta pregunta, dada por la paradoja, es la antinomia: la clase de las clases que no son miembros de sí mismas es miembro de sí misma si y sólo si no es miembro de sí misma. 
no puede decirse que haya habido un esfuerzo persistente y ampliamente difundido por resolver los dos problemas que he mencionado, enfrentándose directa y críticamente con las fuentes de donde surgen. Por la época en que estos problemas se presentaron, había ya fuertes influencias convencionalistas y positivistas trabajando en física y matemáticas; y en esta atmósfera teórica había una inclinación a creer que quizás ahora había llegado para la lógica misma el momento de aceptar el carácter intrínsecamente problemático o convencional de sus conceptos y principios fundamentales. Esto minó el esfuerzo por abordar estos problemas en una forma no comprometedora. En cambio, habia una tendencia a llegar a una especie de paz ad hoc con ellos.

Supóngase, entonces, que hoy se desea volver a enfocar estos problemas y a reconsiderar las posibilidades de su solución. Seguramente se ha aprendido mucho en el medio siglo pasado, desde que estos problemas surgieron, lo cual puede ser una ayuda para esta empresa. Tenemos a nuestra disposición po derosas ideas teóricas nuevas. Además, el análisis que se ha hecho de la estructura de las ciencias empíricas puede servirnos como una especie de guía en este sentido: si vamos a reenfocar estos problemas cruciales de la lógica, necesitamos tener una hipótesis general sobre la estructura de la misma ciencia lógica, de tal manera que podamos juzgar a qué partes o niveles de la ciencia pertenecen estos problemas respectivamente.

Es evidente que no dará resultado el intento de volver a la ingenua concepción de que la lógica formal es una estructura monolítica de verdades a priori. Ciertamente gran parte de la lógica formal ha sido siempre altamente problemática. Pero esto no justifica la conclusión de que la totalidad de la lógica formal tiene un carácter epistemológicamente problemático. Me parece que el error básico ha sido considerar a la lógica formal como monolítica en su estructura, por así decirlo, y, en consecuencia, como epistemológicamente homogénea. Si estamos preparados para renunciar a esta noción, puede ser que encontremos una base para considerar que la lógica formal misma tiene una parte a priori que consiste en certezas intuitivas, $y$ una parte teórica problemática que trata de elucidar estas certezas, sistematizarlas, y desarrollar sus consecuencias suministrando un contexto. formal articulado en el que puedan operar libremente.

Desde este punto de vista, ciertos problemas, como el que se refiere a la consistencia de nuestros conceptos lógicos más intuitivos y fundamentales, nos desafiarán a encontrarles soluciones que vindiquen nuestras certezas intuitivas. Por otra parte, problemas que no amenazan nuestras certezas intuitivas, como el que se refiere a las conectivas proposicionales, pueden considerarse como problemas de elucidación, cuyas soluciones deben satisfacer los criterios de ser lógicamente bien fundados y de tener poder explicativo general. En el presente artículo consideraré estos dos problemas, a los cuales he estado refiriéndome, en el marco de esta hipótesis. 


\section{Primera parte: Las paradojas}

2. Hay ciertas paradojas lógicas que, por medio de un razonamiento autorreferente, derivan contradicciones de premisas que no vemos cómo rechazar y que implican conceptos tales como "clase", "propiedad" y "verdadero". Ahora bien, estos conceptos son cruciales para los principios básicos de la lógica. El punto es, por tanto, casi demasiado claro. Si se ha mostrado válidamente que estos conceptos llevan a contradicción, entonces el uso de estos conceptos dentro de la lógica debe modificarse de forma tal que se prevenga el surgimiento de tal contradicción. Estos mismos conceptos deben modificarse o tratarse de nuevas maneras. Luego, si tales modificaciones no pueden llevarse a cabo sin introducir en los fundamentos de la lógica ideas y axiomas que no son autoevidentes de modo intuitivo, esto pone en duda la verdad a priori de los principios fundamentales de la lógica. La autoevidencia deja de ser un criterio suficiente o necesario para un axioma lógico; y se introducen otros criterios, como el de "naturalidad" y el de poder explicativo. Esto mina entonces la pretensión de que la lógica formal se basa en verdades necesarias y autoevidentes.

Russell, en respuesta a este problema que él mismo fue el primero en formular de un modo general, propuso la "teoría de los tipos" para garantizar la consistencia del razonamiento dentro del sistema de la lógica formal. Esta teoría establece una jerarquía de "tipos" o niveles de clases, por así decirlo, y requiere que un concepto de clase funcione lógicamente sólo en el nivel que le resulte apropiado en la jerarquía. De este modo, una clase no puede ser miembro de sí misma, como sucede en la muy claramente concebida paradoja de las clases, de la que Russell mismo es autor. ${ }^{2}$ Por otro lado, Alfred Tarski desarrolló la teoría de los “metalenguajes" para razonar con consistencia acerca de una proposición o argumento a un nivel lingüístico diferente del de la proposición o argumento mismo. Ésta es una concepción semántica. Su sentido es que, cuando hablamos de propiedades semánticas tales como el significado o el valor de verdad de objetos lingüísticos como las proposiciones o argumentos, no hay garantía de que podamos hacer esto consistentemente dentro del lenguaje a que pertenecen estos objetos lingǘsticos. Para garantizar tal consistencia debemos recurrir a un metalenguaje. 3

Estas teorías jerárquicas, como se les llama, están hechas para evitar que

2 En tanto la teoría de los tipos intenta minimizar el contenido del concepto "clase", sin embargo no lo elimina. $\mathbf{Y}$ al hablar sobre la teoría de los tipos, es natural recurrir a este concepto. -Véase Bertrand Russell, "Mathematical Logic as Based on the Theory of Types", en Russell, Logic and Knowledge, edited by R. C. Marsh, Allen \& Unwin, 1956. Este famoso artículo, que elucida la concepción de las variables de la lógica simbólica, fue publicado por primera vez en 1908 .

3 Véase Alfred Tarski, "The Semantical Conception of Truth". (Una versión en español se encuentra en Antología semántica, compilada por Mario Bunge, Nueva Visión, Buenos Aires.) 
surjan las paradojas. Evitan esto en efecto mediante la exclusión de ciertos usos reflexivos o autorreferentes del lenguaje. Ahora bien, estas teorías dan origen a sus propias dificultades. Introducen gran complicación en la teoría lógica; y cuando se hacen lo suficientemente estrictas para eliminar las contradicciones, tienden a excluir demasiado de nuestro uso intuitivo del lenguaje natural, y de esta manera tienden a ser contraintuitivas. Pero, desde luego, una cosa es no requerir que un principio lógico tenga autoevidencia intuitiva, y otra cosa completamente diferente es permitir un principio que es contraintuitivo, aun si es así sólo en el sentido de que parece irrazonablemente restrictivo. Russell mismo nunca estuvo satisfecho con la teoria de los tipos y siempre insistió en que la consideraba provisional. Mas si la antinomia de Russell fue derivada válidamente, es sin duda necesario algún tipo de modificación de la teoría de las clases para prevenir el surgimiento de la contradicción.

Como se ha dicho, este problema respecto a los fundamentos de la lógica vale retroactivamente para la lógica formal tradicional. Por ejemplo, la antinomia de Russell explota el principio de que poseer la propiedad-condición de membresia de una clase es ser miembro de esa clase, lo cual es la base de la interpretación extensional del razonamiento silogístico. Ciertamente, la antinomia de Russell asumía que una clase es una entidad lógica a la que pueden atribuirse propiedades lógicas; pero ¡cómo puede rechazarse esta asunción sin rechazar el concepto de clase?

Otra de las paradojas que Russell cita al plantear el problema es la vieja paradoja Epiménides, la cual implica el concepto de verdad. Ahora bien, la paradoja Epiménides no lleva a una contradicción estrictamente formal; sin embargo, como se verá, esta paradoja conduce ciertamente a una concepción autocontradictoria. Mientras tanto, la moderna paradoja El mentiroso, desarrollada por Lukasiewicz, implica también el concepto de verdad y deriva una contradicción en la forma de una antinomia. Esta paradoja recurre al propio concepto aristotélico de verdad, y utiliza una forma semántica que Aristóteles mismo formuló. 4

Así resulta claro que, si estas paradojas son derivadas válidamente, es imposible tenerlas a un lado como problemas que son peculiares de desarrollos especiales en la lógica simbólica moderna. Más bien atacan los fundamentos de la lógica formal en cuanto tal. Entonces, con seguridad que era de esperarse que habría un esfuerzo concertado para probar que las derivaciones originales de las paradojas son falaces. Sin embargo, esto no sucedió.

Además esto no puede achacarse completamente al clima intelectual de

4 Ibid. Tarski llama a la forma en cuestión la "forma ( $T$ )" y la formula así: $X$ es verdadera, si y sólo si, $p$, siendo $p$ una oración del lenguaje al que pertenece "verdadero", y siendo $X$ el nombre de $p$. Aunque Tarski atribuye esta forma a Lesniewski, fue usada por Aristóteles en las Categorias, $14^{\mathrm{b}}$ 11 ss. Pero, desde luego, el punto de Tarski, de que esta forma expresa la idea aristotélica de verdad, es perfectamente correcto. 
las primeras décadas del siglo veinte. Parecería que, en general, los filósofos están poco dispuestos a llevar a cabo un cruel análisis que pudiera destruir una buena paradoja. El por qué es esto así constituye un intrigante problema que no intentaré tratar aquí. Sin embargo, me parece que si esto no fuera así, sería incomprensible que durante tantos siglos hayan quedado inexploradas ciertas posibilidades obvias para el análisis de cualquiera de las dos versiones de la paradoja Epiménides. Por esta razón, deseo usar esta paradoja como un ejemplo para mostrar que las derivaciones originales de las paradojas pueden muy bien ser falaces; y que, por tanto, no hay una amenaza irrefutable a la verdad necesaria de nuestros conceptos y principios lógicos intuitivamente autoevidentes.

3. Consideremos primero lo que puede llamarse la versión El mentiroso de la paradoja Epiménides, puesto que implica el concepto "mentira". Se nos dice que Epiménides el cretense dijo: "Todos los cretenses son mentirosos". Interpretando lo que Epiménides dijo como "Todos los enunciados de los cretenses son mentiras", entonces, si este enunciado es verdadero, es falso.

Como lo he mostrado en otra parte, 5 esta paradoja se hace susceptible de análisis en el momento en que se toma en cuenta que una mentira es una aserción intencional que está en función de una creencia. Por ejemplo, si Epiménides afirma "Todos los enunciados de los cretenses son mentiras" con intención de mentir, entonces él cree que este enunciado es falso. Entonces, si esta proposición es no obstante verdadera, lo que puede inferirse es que Epiménides está en un error, mas no se sigue que esta proposición -llamémosla $L$ - es falsa. Esto se aclara si tomamos en cuenta las distintas definiciones posibles de una mentira. Si ésta se define como la falsificación intencional de una creencia verdadera, entonces una aserción mentirosa es falsa. Pero en este caso Epiménides no puede estar mintiendo en este sentido. Porque si $L$ es verdadera y él la cree, para mentir en este sentido no afirmaría $L$, sino $\sim L$. En cambio, si una mentira se define simplemente como la falsificación de una creencia, no se sigue que sea falsa. Según esta definición, si $L$ es verdadera y Epiménides la asevera con intención mentirosa, está mintiendo y su aserción es un caso confirmativo de $L$, pero él está en un error.

Esto dispone de este caso en que la proposición es verdadera, Epiménides cree que es falsa, y la afirma. Hay, sin embargo, cuatro situaciones especificables, determinadas según que la proposición universal sea verdadera o falsa, y según que Epiménides la crea o no. La tercera premisa que determina cada situación es que Epiménides afirma la proposición con intención mentirosa o veraz; así podemos entonces inferir si su intención es mentirosa o veraz.

5 Véase mi artículo, "Belief and Intention in the Epimenides", Philosophy and Phenomenological Research, dic. de 1969 . 
En el artículo mencionado arriba, se analizan los cuatro casos con todo detalle, y no resulta ninguna paradoja. Sin embargo, el análisis tiene un resultado interesante. Resulta que en dos de las situaciones, una de las cuales es existencialmente posible, hay una contradicción en las creencias de Epiménides. Encontramos que la paradoja explota el fenómeno demasiado común de una acción -en este caso una aserción intencional- basada en una creencia que es miembro de un conjunto de creencias contrádictorias. Tales acciones son, desde luego, absurdas; pero esto es un absurdo existencial, no lógico. Tenemos aquí, entonces, un ejemplo de cómo la aparición de una contradicción lógica puede tener su origen en el tratamiento falaz de algún problema no reconocido que está latente en una paradoja.

4. Después de publicado este análisis de la versión del Mentiroso de la paradoja de Epiménides, recibí un comentario crítico de un bien conocido filósofo que me reprochó el haberme metido en un problema que es marginal al problema lógico planteado por las modernas paradojas del Mentiroso, así como por la otra versión de la paradoja de Epiménides. Alegaba que la otra versión, que se basa en una locución de verdad y no implica el concepto "mentira", plantea el clásico problema que pone en duda la consistencia de nuestros conceptos de valor de verdad. ${ }^{6}$

Ahora bien, me parece que hay muy poco que ganar discutiendo sobre cuál de las dos versiones plantea el problema clásico. Mi opinión es que, con anterioridad a la resolución de una paradoja, no podemos juzgar con confianza qué problema plantea. Siempre se pensó que las dos versiones de la paradoja de Epiménides planteaban esencialmente el mismo problema, y Russell citó la versión del Mentiroso. No obstante, el análisis muestra que los problemas implicados en esta versión son la vaguedad del concepto cotidiano "mentira" y el fenómeno de las creencias contradictorias. Pero con seguridad, el problema de la versión del Mentiroso es marginal al problema de la consistencia de nuestros conceptos de valor de verdad. Esto es exactamente lo que he tratado de establecer; y si ahora puede juzgarse que esto es así; ¿no es acaso esto el resultado de mi análisis? La tarea que resta es mostrar que el problema de la versión de la locución de verdad de la paradoja de Epiménides es también marginal a la consistencia de nuestros conceptos de valor de verdad.

La tarea de resolver las paradojas consiste precisamente en mostrar que cada paradoja plantea, a su vez, algún otro problema aparte del que pretende plantear. Ésta es la clave de todo el asunto. Si las paradojas son falaces y no amenazan realmente la consistencia de los conceptos lógicos a que parecen

6 El término "locución de verdad" se usa para una oración de la forma sujeto-predicado que predica de una proposición un valor de verdad. Tales locuciones pueden ser singulares, particulares o universales. Hay desacuerdo entre los filósofos contemporáneos en cuanto a si "verdadero" y "no verdadero" (o "falso") son predicados genuinos. 
dirigirse, entonces es razonable asumir que responden a problemas ocultos cuya concepción aún no es clara, o aun a problemas todavía no descubiertos. Mi hipótesis a este respecto es que la sensibilidad intuitiva del filósofo para tales problemas latentes es la que lo capacita y lo impele a concebir una paradoja filosófica o lógica. No debemos asumir que una paradoja duradera como la de Epiménides o la Antinomia de Russell resultará una pieza trivial de un razonamiento falaz que pueda echarse al cesto. Hay más bien buenas razones para asumir que tales grandes paradojas ocultan auténticos problemas latentes. Así, yo he alegado en otra parte que, en su Antinomia, Russell descubrió una clase de objetos lógicos antes desconocida, pero tenemos que ver que la contradicción de la paradoja es ilusoria, antes de que podamos vislumbrar el problema que Russell atrapó en la red de su razonamiento.?

Paradojas que parecen ser muy similares, como las dos versiones de la de Epiménides, puede ser que cometan falacias bastante diferentes y que planteen problemas de un carácter bien distinto. Por esta razón, no hay un atajo para la solución de las paradojas mediante la buisqueda de soluciones generales de tipos de paradojas que aparentan plantear el mismo problema. Toda paradoja requiere un análisis individual que responda a su particular temática así como a su estructura lógica. Esto hace de la solución de las paradojas una tarea muy laboriosa; pero sospecho que esta tarea es menos laboriosa que la construcción de las complicadas teorías diseñadas para hacer las paces con ellas. Además, si mi enfoque del asunto es correcto, entonces el análisis de cada paradoja tiene su propia recompensa; ya que probablemente sirva para desenterrar y plantear algún problema interesante.

Pero ¿por qué las paradojas de autorreferencia encierran problemas inesperados? Me parece que es así porque, lejos de ser un modo de discurso no digno de confianza, la autorreferencia es un poderoso instrumento de exploración analítica que puede poner a nuestro alcance casos, y aun dominios enteros, que antes pasaban inadvertidos. Por ser un instrumento analítico tan sensible, su uso es peculiarmente susceptible a falacias; y las falacias de una paradoja tienden a colarse justo en el punto donde alguna actitud teórica y no crítica de su autor levanta una barrera que le impide ver el problema latente que la autorreferencia está averiguando, por así decirlo.

Esta manera de ver las paradojas, que he expresado aquí de modo un tanto metafórico, tomó forma para mí en el curso de los análisis de la versión del Mentiroso de la paradoja de Epiménides, de la Antinomia de Russell y de la paradoja de Lukasiewicz, que han aparecido en otra parte. Deseo presentar ahora una especie de caso clínico del trabajo preliminar que he hecho sobre la versión de la locución de verdad de la paradoja de Epiménides como un ejemplo de cómo la autorreferencia puede llevarnos a una

7 Véase mi artículo, "Sobre las paradojas de autorreferencia", en Critica, vol. III, núm. 7/8, enero-mayo de 1969. (Publicado en noviembre, 1970.) 
situación lógico-lingüística que plantea un problema completamente inesperado sobre el uso de las locuciones de verdad. Deseo, sin embargo, hacer hincapié en que el análisis que sigue no pretende ser una solución completa de la paradoja. Se ofrece como una posible base para un tratamiento del problema más estrechamente razonado.

5. Por conveniencia, de aquí en adelante me referiré a la versión del Mentiroso de la paradoja de Epiménides como a Epiménides $(L)$ y a la versión de la locución de verdad como a Epiménides $(T)$. La Epiménides $(T)$ puede enunciarse como sigue:

Epiménides el cretense dijo "Todos los enunciados hechos por cretenses son falsos". Así, si la proposición afirmada por Epiménides es verdadera, vale para sí misma y es falsa.

¿Qué podría ser más simple? ¿Cómo abordarla?

Como ya se ha notado, las dos versiones de Epiménides se consideraban tradicionalmente como enunciados simplemente diferentes de la misma paradoja. El dilema común a ambos se interpretaba así: si Epiménides está diciendo la verdad, está diciendo una falsedad. En tiempos más recientes la interpretación ha recibido un nuevo sesgo. Se arguye que desde el punto de vista de la lógica formal, la paradoja es simplemente una prueba por reductio ad absurdum de la falsedad de la proposición autorreferente en cuestión; pero lo que, no obstante, nos molesta es que esta proposición parece una generalización empirica, mas la prueba de su falsedad necesaria en el caso autorreferente es incompatible con esta manera de verla. Este último punto es el que tenía en mente cuando al principio empecé a trabajar en la paradoja.

Desde luego, el primer paso para el análisis de cualquier paradoja es formular tan claramente como sea posible, al principio, las premisas a que recurre el razonamiento. Así, yo puse:

$(P)$ Todos los enunciados hechos por los cretenses son falsos.

$(Q)$ Epiménides el cretense afirmó $P$.

Ahora bien, en el momento en que separamos y yuxtaponemos estas premisas, vemos que son incompatibles. Sobre la base de lo que explícitamente dicen estas premisas, podemos inferir que si $P$ es verdadera, entonces $Q$ es falsa; porque se sigue de $P$ que Epiménides nunca afirmó un enunciado verdadero. Tenemos, por tanto, Si $P$, entonces no- $Q$. Se sigue entonces lógicamente que, si $Q$, entonces no-P. Además, esta inferencia es justo lo que establece el argumento de la paradoja por la prueba de reductio ad absurdum, que tiene la forma, Si $Q$, entonces $P$ implica no-P. Así, el argumento de la 
paradoja parece ahora no ser sino una prueba válida, indirecta, de la incompatibilidad de las dos premisas del razonamiento. Y si estas dos premisas son ambas empíricas, la paradoja se desvanece.

Pero seguramente el asunto no es tan simple. Ya sabiamos, sobre bases distintas de $Q$, que $P$ es falsa. La cuestión es: ¿por qué $Q$ refuta a $P$ ? Si esto es por la razón lógica de que $Q$ establece el caso autorreferente en que $P$ llega a autorrefutarse, entonces la paradoja realmente no descansa en la incompatibilidad de dos premisas. Porque por $P$ sola, y sobne la asunción de que pertenece al lenguaje hablado por los cretenses, el caso autorreferente surge y no puede ser excluido. Además, el caso autorreferente implica una genuina contradicción. Porque es fácil ver que, en este caso, fla falsedad de $P$ es a uno y al mismo tiempo, una instancia confirmadora de $P$ que la refuta!

Durante más de dos años después de abordar la relación entre $P$ y $Q$, no encontré ninguna manera de llevar el análisis adelante. Luego un día, en un momento de profunda irritación con todo el problema, pensé: ¡Bien! si la autorreferencia es lo que está en cuestión, consideremos el problema en un contexto que implique el caso autorreferente de la manera más clara y necesaria. Así, en una hoja de papel limpia, escribí la proposición (que llamaré $R$ ),

\section{Todos los enunciados son falsos.}

Pocas horas después, había elaborado el tratamiento de la paradoja que se presenta a continuación.

Debe echarse una mirada fresca a $R$. ¿Qué significa $R$ ?, cacaso no tiene exactamente el mismo tipo de significado que el Principio de Tercero Excluido? Ciertamente sí. $R$ afirma que todos los enunciados tienen el mismo valor de verdad, a saber, "falso". Así, con respecto a cualquier discurso o lenguaje para el que $R$ es verdadera, jla lógica asumida es univalente! En este lenguaje no puede haber negación. Además, $R$ tendría la misma significación a este respecto si el valor de verdad que predicara fuese "verdadero". Pero, dado que el valor predicado es "falso", esto tiene la consecuencia adicional de que el caso autorreferente es imposible; porque para un lenguaje para el que $R$ es verdadera, no puede afirmarse dentro de dicho lenguaje ninguna ley o descripción verdadera de ese lenguaje iprecisamente porque es verdadera!

De este modo, el problema se transforma totalmente.

Desde luego, es imposible construir un lenguaje artificial cuyos enunciados deban tener todos el mismo valor de verdad. ¡No se puede construir una lógica univalente amputando un valor de verdad en la forma en que Abbott construyó su Flatland bidimensional amputando una dimensión espacial! La misma noción de tal construcción es lógicamente absurda. Sin embargo, como veremos en un momento, esto es justamente lo absurdo implícito en Epiménides $(T)$. 
Ahora vemos que para tratar a $R$ como un enunciado sobre un lenguaje $L$, ciertamente debemos recurrir a la noción de metalenguaje. Reformulemos $R$ así: "Todos los enunciados que pertenecen a $L$ son falsos." Afirmamos luego $R$ en un lenguaje natural $N$ cuyo uso está regido por una lógica bivalente. Puede expresarse esta situación escribiendo

$\left(R_{N}\right)$ Todos los enunciados que pertenecen a $L$ son falsos,

siendo $R_{N}, R$ afirmada en $N$. Es claro que, en esta situación, el caso autorreferente no puede surgir si $R$ no pertenece también a $L$; y si $R$ es verdadera, $R$ no pertenece a $L$. Tenemos, pues: si $R$ es verdadera, el caso autorreferente no puede surgir. Se sigue entonces que cualquier proposición que implique que $R$ pertenece a $L$ es incompatible con $R$.

Volvamos ahora a Epiménides $(T)$. ¿Hay alguna diferencia esencial entre $R_{N}$ y $P$ ? Ciertamente no. Si sustituimos " $L$ " en $R_{N}$ por "el discurso de los cretenses", tenemos entonces exactamente la misma situación lógica-lingüística. Podemos escribir,

$\left(S_{N}\right)$ Todos los enunciados que pertenecen al discurso de los cretenses son falsos.

La única diferencia entre $R_{N}$ y $S_{N}$ es que la última especifica que el lenguaje en cuestión es el de cierta gente; y al especificar que es el lenguaje de los cretenses, que son un pueblo real, creamos la ilusión de que el lenguaje en cuestión es un lenguaje natural cuyo uso está regido por una lógica bivalente. Ésta es la razón por la cual el significado de $P$, que es únicamente una manera vaga de expresar $S$, nos ha eludido.

Supongamos que se objeta que $P$ no implica necesariamente la existencia de un lenguaje cretense. Por ejemplo, los cretenses podrian hablar lenguajes naturales diferentes. Sin embargo, en el momento en que asumamos que los cretenses se comunican unos con otros, entonces, por $P$, habría una especie de lenguaje cretense híbrido en el que, por ejemplo, no hay negación. En efecto, no importa cuáles pudieran ser las circunstancias existenciales, si un cretense tiene un lenguaje -y seguramente esto se asume en $P$-, entonces, por $P$, se rige por una lógica univalente. No hay manera de eludir esto.

En $S$, he modificado la formulación de $P$ para hacer ver que su tema es lingüístico. La diferencia entre las dos versiones de la paradoja de Epiménides depende justamente de este punto. La versión del Mentiroso se ocupa de una proposición universal genuinamente empírica en relación con otras pro posiciones empíricas, y llega a implicar un absurdo existencial. "Todos los enunciados hechos por los cretenses son mentiras" versa sobre la intención mentirosa o veraz con que se afirman los enunciados. Además, la versión del 
Mentiroso supone una lógica bivalente, puesto que de ella depende el mismo concepto de mentira. En efecto, lo que es comun a las dos paradojas es que en sus premisas está en juego una lógica bivalente. Pero mientras la versión del Mentiroso tácitamente la asume para abordar una cuestión empírica, la $P$ de la versión de la locución de verdad es ella misma una premisa lógica que la niega. Esta premisa $P$ es lógicamente falsa porque atribuye a un supuesto lenguaje una propiedad que es incompatible con el carácter lógico del lenguaje. Seguramente, hay aquí mucho espacio para el análisis del sentido preciso en que $P$ es lógicamente absurdo. Sin embargo, está fuera de duda que asi es.

Estamos ahora listos para reconsiderar el argumento inicial sobre la relación entre $P$ y $Q$ que originalmente se desarrolló sobre la errónea asunción de que sólo está en juego un lenguaje. Al reconsiderar el argumento, debemos mantener cierta reserva al considerar la verdad de $P$. También, puesto que el argumento asume una lógica bivalente, podemos especificar que lo realizaremos en nuestro lenguaje natural $N$, a diferencia del lenguaje cretense $C$. Finalmente, podemos estipular que si un enunciado es hecho por un cretense, pertenece al lenguaje $C$. Entonces, a fin de discutir la relación entre ellas, podemos formular como sigue las premisas de la paradoja:

$\left(P^{\prime}{ }_{N}\right)$ Todos los enunciados que pertenecen a $C$ son falsos.

$\left(Q^{\prime}{ }_{N}\right)$ Epiménides el cretense afirmó $P^{\prime}$.

Es claro que si $P^{\prime}$ es verdadera, no pertenece a $C$. Pero $Q^{\prime}$ implica que $P$ pertenece a $C$. Por tanto, si $P^{\prime}$ es verdadera, $Q^{\prime}$ es falsa. Pero si $Q^{\prime}$ es verdadera y $P^{\prime}$ pertenece a $C$, entonces $P^{\prime}$ se autorrefuta.

Lo que este argumento prueba es que no es el caso que $P^{\prime}$ sea verdadera $y$ pertenezca a $C$. No prueba que $P^{\prime}$ sea necesariamente falsa, sino solamente que el lenguaje artificial descrito por $P^{\prime}$ no puede contener a $P^{\prime \prime}$. El caso autorreferente sirve para probar la inconsistencia de la concepción de que $P^{\prime}$ es verdadera y pertenece a $C$. Además, esta prueba depende de $Q^{\prime}$; porque $P^{\prime}{ }_{N}$ por sí misma no pertenece a $C$.

Desde luego, no habrá que llevar demasiado lejos esta absurda situación. Lo importante es ver que en este nuevo enfoque analítico de la paradoja, ésta se hace susceptible de solución en una forma que cambia completamente el juego sobre las críticas del lenguaje natural.

6. ¿Cuál es, entonces, el problema planteado por Epiménides (T)? Parecería que el uso de locuciones de verdad universales está expuesto a la muy peculiar falacia de implicar la existencia de un lenguaje artificial con un solo valor de verdad. Éste es seguramente el problema central planteado por la paradoja. Entonces, puesto que, como es bien sabido, el mal uso de las locu- 
ciones de verdad puede llevar también a otros tipos de anomalías, hay mucho qué hacer para clarificar todo el tema del uso correcto de tales locuciones. No cabe duda de que, como la autorreferencia misma, ellas se prestan también a ser usadas falazmente. Hay otra falacia, o sea, el error categorial, a la cual está expuesto su uso; y parece seguro conjeturar que el análisis de las paradojas restantes revelará otras.

Mientras tanto, me parece que las consideraciones aquí aducidas respecto a la paradoja de Epiménides proporcionan por sí mismas una base suficiente para la seria consideración de la hipótesis de que las derivaciones originales de las paradojas lógicas son falaces. Porque seguramente no hay otra paradoja que pueda compararse con la Epiménides $(T)$ en el sentido de que combina tan completamente un carácter estrictamente lógico con la sencillez de concepción y la validez de su razonamiento. Todavía aun este problema nos reserva sorpresas cuando forzamos el tema. Además, encontramos que precisamente cuando ponemos en juego, de la manera más clara posible, el aspecto autorreferente del problema, entonces éste se hace susceptible de análisis.

Como lo he mostrado en otra parte, las derivaciones de la Antinomia de Russell y la del Mentiroso, de Lukasiewicz, se pueden interpretar como falaces. ${ }^{8}$ Esto reforzó mi propia convicción de que las paradojas tienen solución; pero no fue sino hasta que la versión de la locución de verdad de la paradoja de Epiménides se abrió al análisis plenamente, por así decirlo, y lo hizo en forma tan dramática, que me pareció que: hay ahora suficientes bases objetivas para asumir, como una hipótesis de trabajo de la lógica formal, que las paradojas son falaces y que las teorias jerárquicas no son esenciales para salvaguardar de consecuencias contradictorias a los principios fundamentales de la lógica.

Esta conclusión no implica necesariamente que sean superfluas las teorías que se han desarrollado para hacer las paces con las paradojas. Es innegable que las ideas teóricas surgidas en este contexto pueden desempeñar un papel muy importante al nivel de la construcción de teoría lógica, lo cual se discutirá en la segunda parte de este artículo. Se ha visto cómo surgió la noción de metalenguaje, de una manera natural, en el curso del análisis de la paradoja Epiménides $(T)$. De nuevo, es evidente que la noción de Russell de los tipos lógicos es altamente relevante para la concepción teórica de las variables de la lógica simbólica.

Mucho menos puede concluirse que las paradojas son de escasa importancia teórica, y que pueden ser ignoradas. Nuestra confianza en los fundamentos de la lógica debería más bien expresarse en un esfuerzo concertado y crítico por resolver las paradojas en una forma completamente satisfactoria. Indudablemente esto enriquecerá la problemática de la lógica formal trayen-

8 Ibid. 
do a luz los problemas imprevistos que son atrapados en las redes de las paradojas.

Hay una razón para el surgimiento de paradojas en búsquedas intelectuales. Es uno de los modos en que la vaga intuición de problemas latentes los atrapa.

Segunda parte: Las relaciones-funciones de verdad

7. Aun cuando la certeza de los principios fundamentales de la lógica puede llegar a ser plenamente vindicada, esto mismo no puede, sin embargo, apaciguar la duda filosófica, hoy tan extendida, respecto a la fuerza lógica, por así decirlo, de la lógica simbólica misma. ¿Es la lógica simbólica meramente un cálculo consistente que recorre su propio camino según sus propias reglas, o es que se gobierna realmente por un concepto de inferencia lógica estricta? Esta pregunta ha surgido como respuesta a la llamada "interpretación material" de las conectivas proposicionales de la lógica simbólica.

Si hemos de enfrentarnos a este problema, debemos considerar cómo surgió la interpretación material y en qué consiste. Pero primero consideremos qué clase de problema lógico está en juego aquí.

Una conectiva proposicional como "y", "o", y "si... entonces..." expresa una relación lógica entre proposiciones; y la lógica simbólica parte de la suposición de que tal relación se caracteriza por lo que puede llamarse un conjunto de condiciones de verdad. Ahora bien, la noción de que una relación entre proposiciones se expresa por cómo el valor de verdad de una proposición afecta al valor de verdad de la otra, se remonta a Aristóteles. La lógica simbólica ha desarrollado y generalizado esta noción. Ha introducido formas "funciones de verdad", y con ellas cierta interpretación de su significado lógico. Al hacer esto, la lógica simbólica ha elaborado una construcción teórica. Si, por tanto, no estamos satisfechos con esta construcción, y asumo esto, necesitamos tener alguna noción general de las construcciones lógicas teóricas, por medio de la cual podamos orientar nuestra crítica de ella.

La lógica abunda en conceptos construidos y formas definidas que se introducen para proveer de un contexto sistemático y formal en el que nuestros principios lógicos fundamentales y nuestras certezas inferenciales puedan tener el papel más libre y las consecuencias más ricas posibles. La generalización de la lógica consiste precisamente en construir sistemas articulados de formas que ensanchan el campo de operación, por así decirlo, de nuestras certezas lógicas. Tales construcciones entrañan los conceptos básicos que entran en nuestras certezas lógicas; sin embargo, su propia corrección y utilidad lógicas tienen que ser probadas. Además, aun cuando hayan resistido las más rigurosas pruebas, no puede pretenderse que sean lógicamente necesarias. Por ejemplo, ¿quién se aventurará a decir que la reconstrucción de Russell de las 
formas lógicas de las proposiciones categóricas es lógicamente necesaria y no tiene alternativa? Y sin embargo es una de las piedras angulares del cálculo proposicional de la lógica simbólica. Es simple, conceptualmente fundada, y está bellamente sincronizada con las formas generales de este cálculo.

¿Cuáles son, entonces, los requisitos teóricos para tales construcciones lógicas? Para los efectos de la presente discusión, deseo simplemente sugerir al respecto, grosso modo, algunas consideraciones preliminares.

Primero, la construcción debe ser no sólo compatible con nuestras certezas inferenciales, sino que debe ser congruente con ellas en un sentido muy especial. Una forma lógica puede prestarse como un medio para la operación de inferencia formal sólo sobre la base de su propio significado lógico. Debe, por tanto, tener suficiente sentido lógico para fundamentar las inferencias que permite.

Segundo, la construcción debe encajar, en una manera sincronizada, en el sistema a que pertenece.

Tercero, una construcción lógica debe ser consistente, no sólo en el sentido de que no conduzca a contradicción, sino también en el sentido de que no contenga ambigüedad.

Se podría querer añadir a esta lista el que una construcción lógica no deba concebirse de tal forma que implique asunciones no lógicas, o algo de este estilo. Sin embargo, me parece que se ganaría poco orientando la crítica de la interpretación material hacia esta condición, la cual ciertamente está en juego, pero por sí misma no sirve para enfocar nuestra atención sobre los problemas estrictamente lógicos que están en juego.

Volvamos ahorá a la problemática a la cual responde la interpretación material de las relaciones-funciones de verdad. Deseo considerar esta problemática en el contexto de su desarrollo histórico, de modo que podamos apreciar todo el alcance de la generalización de la lógica lograda en la lógica simbólica de Russell y Whitehead. Luego, si desde este punto de vista encontramos que este sistema de lógica tiene alguna debilidad teórica, lejos de sentirnos impelidos a menospreciarlo, nuestro impulso será considerar por qué medios puede superarse tal debilidad. Como he sugerido en el caso de las paradojas, es cuestión de dirigir nuestra atención a las fuentes del problema. Si hacemos esto, quizá podamos al menos empezar a vislumbrar la dirección en que puede encontrarse la solución del problema.

8. Aristóteles fue el que primero concibió las relaciones lógicas entre las formas categóricas en términos de cómo el valor de verdad de una proposición con una forma afecta al de una proposición con otra forma. A este respecto Aristóteles, en De Interpretatione, estaba explícita y principalmente interesado en la diferencia entre las relaciones de contradicción y contrarie- 
dad. Especificó claramente los respectivos conjuntos de condiciones de verdad, de contradicción y contrariedad, y usó también este enfoque para otras relaciones entre las cuatro formas. Más tarde, en el Cuadrado de Oposición tradicional, todas las relaciones que se dan entre las formas categóricas fueron especificadas en términos de sus condiciones de verdad. No hay, entonces, nada más tradicional en la lógica formal que este tipo rudimentario de enfoque de función de verdad de las relaciones entre proposiciones. Desde luego, las relaciones entre las proposiciones categóricas se concibieron al mismo tiempo como lógicamente necesarias.

Por otro lado, y también desde sus orígenes, la lógica tradicional se interesó en las formas conjuntiva, condicional y disyuntiva, por cuyos medios las proposiciones se relacionan entre sí en las proposiciones compuestas. Ahora bien, las proposiciones compuestas, formadas por medio de conectivas como " $y$ ", . o", y "si... entonces...", comúnmente se usan para relacionar proposiciones que pueden o no estar relacionadas entre sí analíticamente. Cuando usamos formas compuestas de este tipo, asumimos, con base en la significación de la conectiva, una conexión lógica entre las proposiciones así relacionadas que justifica ciertos tipos de inferencia. Considérese este ejemplo de implicación que, según recuerdo, es de Aristóteles: "Si llueve, entonces el suelo se moja". Intuitivamente sentimos que éste es un uso justificado de la conectiva "si... entonces..."; así, dado que llueve, no vacilamos en inferir, por modus ponens, que el suelo se estrá mojando. Análogamente para la conectiva disyuntiva "o", cuando su uso se nos presenta como válido, dada la falsedad de uno de los disyuntos, inferimos la verdad del otro. Este tipo de inferencia es el que da al razonamiento cotidiano su gran flexibilidad.

Ahora bien, parece natural considerar que podrian sintetizarse de alguna manera estos dos enfoques de las relaciones entre proposiciones. Es claro desde el principio que la forma "si... entonces..." tiene las mismas condiciones de verdad que la relación de implicación del Cuadrado de Oposición tradicional; y "o", cuando se interpreta como disyunción inclusiva, tiene las mismas condiciones de verdad que la subcontrariedad. ¿No sería entonces posible construir un sistema de formas, basado sobre tales conectivas definidas en términos de sus condiciones de verdad, que tenga el poder lógico de expresar todas las relaciones lógicas fundamentales entre proposiciones?

La lógica tradicional no trabajó en esta dirección, pero ésta era la intuición que gradualmente se hizo más clara cuando la lógica matemática comenzó a desarrollarse a mediados del siglo xrx. El avance teórico decisivo, que apuntó hacia la posibilidad de tal síntesis formal, fue el descubrimiento de la correspondencia precisa entre el cálculo algebraico de clases y un cálculo proposicional, éste basado en una interpretación proposicional de los axiomas del primero, siendo las conectivas conjuntiva y disyuntiva del último 
lógicamente análogas al producto y suma lógicos del primero. Este descubrimiento fundamentó la hipótesis de que sería generalmente válido un cálculo proposicional generalizado, construido sobre estas conectivas básicas. Tal cálculo aportaría un sistema completamente general de deducción que serviría como base de la lógica de las funciones proposicionales, de las relaciones, y de las clases; y sería capaz de explotar y desarrollar para todos estos usos lógicos la flexibilidad y poder que, de una manera rudimentaria, caracteriza al uso de las conectivas proposicionales en el lenguaje ordinario.

Esta gran concepción generalizadora encontró su primera expresión completa y, al mismo tiempo, su realización, en Principia Mathematica de Russell y Whitehead. Nada puede menguar la importancia histórica de esta hazaña. Si tiene defectos, esto es de esperarse. Esto no es un motivo para menospreciar el logro, sino más bien un desafío para intentar contribuir al mejoramiento de la teoría.

Desde De Morgan y Boole en adelante, quienes habian contribuido más al desarrollo de la lógica simbólica fueron matemáticos. Más tarde, aquellos cuyo trabajo fue más decisivo para el surgimiento de la concepción del cálculo proposicional general fueron, por así decirlo, filósofos-matemáticos, cuyo interés dominante estaba en los fundamentos lógicos de las matemáticas. Pero no debe permitirse que esta circunstancia histórica oscurezca el hecho de que este cálculo proposicional es una síntesis que surgió de ideas originarias, contenidas en la lógica tradicional, y las desarrolló. Que este desarrollo pudo ser llevado a cabo sólo por matemáticos que vieron la analogía entre las operaciones del álgebra y las de la lógica proposicional, no hace a la lógica simbólica menos lógica. Además, el problema central que subsiste, a saber, el de la interpretación de las conectivas proposicionales, es estrictamente lógico. Consideremos ahora cómo surgió este problema.

La nueva lógica tenía que responder a la pregunta ¿sobre qué bases puede considerarse verdadera o falsa una proposición compuesta? Desde luego, esto era equivalente a la pregunta: ¿cuál es el sentido lógico de una conectiva como " $y$ ", "o", y "si... entonces..." que es afirmada, con reclamación de su verdad, por una proposición como "si llueve, entonces el suelo se moja"? La concepción de las relaciones del Cuadrado de Oposición tradicional no podía proporcionar una respuesta a esse problema por dos razones. Primero, estas relaciones son analíticas y se conciben como lógicamente necesarias, en tanto que las formas proposicionales generales deben poder usarse para expresar proposiciones empíricas como "Si llueve, entonces el suelo se moja", o "Si Juan no asistió a la junta, entonces o estaba enfermo o estaba fuera de la ciudad."

Considérese la última proposición. Si Juan no estaba enfermo ni fuera de la ciudad, ni asistió a la junta, intuitivamente sentimos que esta proposición es falsa. Es claro que el valor de verdad de la proposición compuesta 
depende en algún sentido de los valores de verdad de las proposiciones componentes. Pero ¿en qué sentido? Esto nos lleva a la segunda inadecuación del Cuadrado tradicional. La concepción del valor de verdad de una proposición compuesta que expresa una relación como implicación o contrariedad, etc., entre dos proposiciones, no entra del todo en el Cuadrado. Los únicos valores de verdad considerados en el Cuadrado son los de dos proposiciones separadas, $p$ y $q$. Pero la nueva lógica pone en juego la forma de implicación " $p \supset q$ " (léase "si p, entonces q" o " $p$ implica q"); y esta for$\mathrm{ma}$, en líneas de razonamiento, entre en relación con otras formas de este tipo, como "p v q" (léase "p o q", en el sentido de disyunción inclusiva). Llegó a ser crucial, por tanto, el ser factible considerar los valores de verdad de proposiciones con tales formas. ¿Cómo había de hacerse esto?

Necesitamos tener un ejemplo en mente; tomemos, pues, la relación de implicación. Recordando sus condiciones de verdad, tenemos: la verdad de $p$ determina la verdad de $q$, y la falsedad de $q$ determina que $p$ es falsa; pero la verdad de $q$ deja a $p$ indeterminada, y la falsedad de $p$ deja a $q$ indeterminada. Entonces, dados los cuatro posibles conjuntos de valores de verdad de cualquier par (p, q), ${ }^{9}$ el único caso que se excluye por esta relación es aquel en que $p$ es verdadera y $q$ es falsa. Las condiciones de verdad de esta relación se expresan, por tanto, por la fórmula: "No es el caso que $p$ es verdadera y $q$ falsa."

Puesto que estas condiciones de verdad expresan la relación entre las formas categóricas (A, I), podemos decir: "Si A, entonces I." Además hay otras relaciones, como la relación lógica de inclusión de clases y la relación existencial de causalidad suficiente, que tienen estas mismas condiciones de verdad. En efecto, la propia relación de deducción lógica tiene estas mismas condiciones de verdad. Así, para caracterizar a un argumento deductivo válido, decimos comúnmente en lógica: "No es el caso que las premisas son verdaderas y la conclusión es falsa." Este enunciado es lógicamente equivalente a la forma implicativa de la fórmula deductiva: "Si las premisas son verdaderas, entonces la conclusión es verdadera."

¿Qué significa, entonces, "si. . . entonces..."?

El problema aquí es lograr un concepto preciso de una forma lógica, y este concepto debe satisfacer ciertas condiciones. Primero, el significado de la forma debe ser tal que sirva como la expresión lógica de cualquier relación que sea caracterizada por las condiciones de verdad de esta forma. Segundo, este significado ha de tener un carácter general tal que varíe sistemáticamente de acuerdo con las variaciones en las condiciones de verdad del conjunto entero de formas compuestas. Por ejemplo, acabamos de ver que " $p \supset q$ " es lógicamente equivalente a " $(p \cdot \sim q)$ " (leyéndose " " y ".",

9 Estos son, en el orden convencional: P-verdadera, Q-verdadera; P-verdadera, Q-falsa; P-falsa, Q-verdadera; P-falsa, Q-falsa. 
"no" y "y" respectivamente). Pero la forma conyuntiva "p - q" tiene un conjunto de condiciones de verdad muy diferente del de la implicación. Así pues, si esta segunda condición no fuese satisfecha, no podría haber un único criterio según el cual las proposiciones, en estas formas diferentes, fuesen verdaderas o falsas. ${ }^{10}$ Es fácil ver que todo el sistema de inferencia de la lógica simbólica depende de que haya un único criterio para la verdad de una proposición compuesta.

Enfrentados a este difícil problema, Russell y Whitehead adoptaron la siguiente solución: las formas " $\mathrm{p} \supset \mathrm{q}$ " " $\mathrm{p}$ v q", etc., deben considerarse como funciones de verdad en un sentido que ellos propugnaron muy preciso. El significado de la forma de una función de verdad está contenido en la fórmula que establece qué conjuntos de valores de verdad son permitidos o excluidos por la forma. El significado de la forma implicativa es: "No es el caso que $p$ es verdadera y $q$ es falsa." Para la forma disyuntiva, " $p$ v q", tenemos, " $p$ y $q$ no son ambas falsas". $\mathrm{Y}$ así para las demás.11 Se sigue entonces $\longrightarrow$ al menos así se pretende- que una proposición en la forma de función de verdad es verdadera si los valores de verdad de $p$ y $q$ no son excluidos por la fórmula que expresa las condiciones de verdad de la función en cuestión. Así resulta que una proposición implicativa es verdadera siempre que $p$ sea falsa y siempre que $q$ sea verdadera.

Éste es, entonces, el meollo de la interpretación material, que hace que el valor de verdad de una proposición con forma de función de verdad dependa solamente de los valores de verdad de las proposiciones componentes, con independencia total de cualquier otra consideración, sea cual fuere ésta.

9. Si hemos de tener una fundada actitud crítica hacia la interpretación material, no debemos estar demasiado impresionados por las aparentes anomalías por las que se culpa comúnmente a esta interpretación. Por ejemplo, se aduce con frecuencia que la interpretación material implica la verdad de una implicación sin base como "Si la tierra es esférica, entonces Bruto apuñaló a César." Ahora bien, no me parece que esta objeción tenga fundamento. $\mathrm{Ni}$ es una simple cuestión de que ordinariamente no usamos las formas de las funciones de verdad de esta manera que carece de base, como tan débilmente lo han mantenido los mismos defensores de la interpretación material.

10 Debe tenerse en cuenta que la función negativa es una función de contradicción. Asf " $\sim$ p" equivale a "p es falsa".

11 Hay 16 conjuntos especificables de condiciones de verdad para un par de proposiciones $(p, q)$, que determinan las 16 formas básicas de funciones de verdad, siendo catorce de éstas contingentes. Todas las funciones de verdad pueden expresarse simbólicamente en una forma muy simple con el uso de las conectivas "." "v", y " $\supset$ ", combinadas con la función negativa " $\sim$ ". Para el ahora clásico análisis de estas formas por medio de "tablas de verdad", véase el Tractatus Logico-Philosophicus de Wittgenstein, 4.31-4.442, y 5.101. -Todo este tema se discute posteriormente abajo, en la sec. 10. 
Ni el sentido común ni el conocimiento científico de ninguna época histórica particular pueden servir como criterio para el uso permisible de las formas de las funciones de verdad. Tal criterio sólo puede ser proporcionado por una fundada concepción lógica de ellas, concepción que, al mismo tiempo, permite el uso más libre posible de ellas para los propósitos de la hipótesis y el descubrimiento científicos. Bien puede ser que el criterio material de verdad sea inaceptable como criterio de verdad. Pero de esto no se sigue que una reinterpretación lógicamente aceptable de la formas de las funciones de verdad deba excluir los usos de ellas que parezcan absurdos para el sentido común. Hay un juego lógico al que se puede recurrir para ilustrar por qué es esto así. Comenzando con una implicación aceptable al sentido común, del tipo no-analítico, que ocurre en la conversación diaria, constrúyase una cadena de tales implicaciones, de tal manera que el consecuente de la primera se haga antecedente de la segunda, y así sucesivamente. Se encontrará que pueden formarse algunas implicaciones que suenen muy raras del antecedente de la primera y el consecuente de algún miembro $n$ de la cadena. ¿Invalida esto el uso de acuerdo con el sentido común, de la forma implicativa? Ciertamente no. El principio que está aquí en juego es que la lógica debe asumir una especie de interrelación universal de las cosas, precisamente como lo hace la ciencia empírica. Al usar la lógica, ordinariamente dirigimos nuestro razonamiento a aquellas conexiones que parecen en algún sentido cercanas o inmediatas. Pero no hay ningún principio lógico que nos prohiba dirigir nuestro razonamiento a conexiones más rebuscadas. Por esta razón, no me ocuparé de aquí en adelante de este tipo de objeción a la interpretación material.

10. Me parece que el mejor punto de partida para la crítica de la interpretación material se ofrece por la consideración de cómo opera ésta en el contexto del conjunto entero de las formas de las funciones de verdad. Wittgenstein mostró que hay dieciséis conjuntos posibles de condiciones de verdad para todo par de proposiciones $(p, q)$; y de ellas, catorce determinan formas lógicamente contingentes cuyas instanciaciones pueden ser verdaderas o falsas. Ahora bien, hay cuatro posibles conjuntos de valores de verdad para todo par ( $p, q)$, esto es: p-verdadiera y q-verdadera; p-verdadera y q-falsa; p-falsa y q-verdadera; y p-falsa y q-falsa. Cada conjunto de condiciones de verdad excluye o incluye como una alternativa, cada uno de los cuatro conjuntos de valores de verdad. Por ejemplo, considérese la forma implicativa:

$\begin{array}{ccc}\mathrm{p} & \mathrm{q} & \\ \mathrm{T} & \mathrm{T} & \text { incluido } \\ \mathrm{T} & \mathrm{F} & \text { excluido } \\ \mathrm{F} & \mathrm{T} & \text { incluido } \\ \mathrm{F} & \mathrm{F} & \text { incluido }\end{array}$


Es claro que esta tabla corresponde en algún sentido a la fórmula: "No es el caso que $p$ es verdadera y $q$ es falsa."

La situación general entonces es que cada conjunto de valores de verdad está incluido como una alternativa por siete de las formas contingentes, y está excluido por las otras siete. Entonces, por el criterio material de verdad, para algún par dado de proposiciones $(P, Q)$, si tenemos P-verdadera y $Q$-verdadera, esto nos permite considerar como verdaderas a " $P \supset Q$ ", “ $P$ v $Q$ ", "P • $Q$ ", " $(\mathbf{P} \supset \mathbf{Q}) \cdot(\mathrm{Q} \supset \mathrm{P})$ "; y así sucesivamente para las otras tres formas contingentes, cuyas condiciones de verdad incluyen P-verdadera y Q-verdadera como una alternativa.

Mas cuando aseveramos "P $\supset Q$ ", seguramente queremos decir que todo el conjunto de condiciones de verdad de la implicación vale para el par $(P, Q)$; y esto no se confirma por el simple hecho de que $P$ y $Q$ son ambas verdaderas. Todo lo que se comprueba por el hecho de que $\mathrm{P}$ y $Q$ son ambas verdaderas es que "P $\supset Q$ " no ha sido refutada.

Demos al llamado criterio de verdad de la interpretación material el nuevo nombre, el criterio unilineal, por referencia a una tabla como la de arriba. Entonces podemos decir que el criterio unilineal simplemente no es un criterio de verdad que responda al pleno sentido lógico de las formas de las funciones de verdad. Las inferencias permitidas por una forma tal dependen de todo el conjunto de sus condiciones de verdad, ${ }^{12}$ sin embargo no está confirmado sobre la base del criterio unilineal que cualquier conjunto de valores de verdad en su totalidad vale para un par dado de proposiciones (P. Q). De esta manera vemos que el criterio unilineal de por si no garantiza la verdad de justamente esa significación lógica que da a las formas de las funciones de verdad su poder inferencial.

Hay, entonces, una ambigüedad fundamental en la interpretación material misma; porque ésta considera a las funciones de verdad como dotadas de un poder lógico que no está confirmado por su llamado criterio de verdad. Esto significa que la interpretación material no satisface el tercer requisito para una construcción lógica, porque no es plenamente consistente en un sentido adecuado.

Intentemos seguir la pista a la fuente de esta inconsistencia de la interpretación material. A este respecto me parece que nos aguarda una verdadera sorpresa.

Los partidarios de la interpretación material insisten constantemente en que todo el significado de una forma-función de verdad está dado por la fórmula que expresa sus condiciones de verdad. Entonces, mi tesis es que estas

12 O sea, el conjunto de sus condiciones de verdad es la base de las inferencias que la forma permite o no. Ésta es la razón por la cual podemos usar tales formas sin conocer los valores de verdad que de hecho tengan las proposiciones componentes. Es cuestion de cómo el valor de verdad de una afecta al de otra; y esto es lo expresado en un conjunto de condiciones de verdad. 
fórmulas mismas son inherentemente ambiguas. Son una contrapartida lingüistica de las figuras Gestalt alternantes de los sicólogos. ${ }^{13}$ Considérese la fórmula, $p$ y $q$ no son ambas falsas. Cuando fijamos nuestra atención en esta fórmula, por un momento parece significar que todo el conjunto de alternativas permitidas por la relación de disyunción vale para el par (p, q). Pero un momento después parece significar solamente que $p$ y $q$ no tienen cierta combinación de valores de verdad. En esta alternación de significado, la primera interpretación está en el contexto lógico en que la fórmula es lógicamente equivalente al enunciado íntegro de las condiciones de verdad de la disyunción inclusiva, en tanto que la segunda es una interpretación literal, verbal. Ahora bien, el criterio unilineal se basa en la interpretación literal; ipero el poder lógico de las funciones de verdad está concebido en términos de la interpretación lógica!

Con seguridad debe ser ésta la fuente de la discrepancia tan molesta entre el poder lógico del cálculo proposicional de la lógica simbólica, y la debilidad lógica de su interpretación material. Porque la interpretación material no reconoce las bases de la fuerza lógica de las funciones de verdad lo que, sin embargo, damos por entendido.

11. Si este análisis es correcto, entonces no puede decirse que la concepción de las funciones de verdad de la lógica simbólica no esté lógicamente fundamentada. Dentro del contexto de las posibilidades lógicas, la noción de un conjunto de alternativas, que Boole introdujo en la teoría básica de la lógica moderna, se adecúa para fundamentar el poder lógico de las funciones de verdad; y ésta es la noción expresada en las fórmulas lógicas de dichas funciones y que se hace explícita en sus tablas de verdad. Lo que, sin embargo, puede decirse, es que el permitir que el criterio unilineal funcione como criterio de verdad, tiende a viciar el fundamento lógico de las funciones de verdad. Ésta es, entonces, la ambigüedad inherente a la interpretación material misma. Así, parece razonable considerar que el problema de superar la interpretación material consiste en cómo eliminar esta ambigüedad.

Supóngase, entonces, que para tener una hipótesis de trabajo se formulan los dos puntos siguientes:

(1) El sentido lógico básico de una forma de función de verdad es que cierto conjunto de condiciones de verdad vale por un par de proposiciones $(p, q)$. Entonces una proposición en la forma de cierta función de verdad es verdadera, cuando el conjunto de condiciones de verdad que corresponde a esa forma, vale efectivamente para sus proposiciones componentes $(p, q)$. Ahora bien, esto no puede juzgarse sobre la base del criterio unilineal. Así, si con-

13 Para una recientè e interesante discusión de las figuras Gestalt, véase Fred Attneave, "Multistability in Perception", Scientific American, dic. de 1971. Que hay una contrapartida lingüistica de este fenómeno, es ello mismo sugerente. 
cedemos, como parece ineludiblemente necesario, que no hay otro criterio de verdad disponible para los propósitos formales del cálculo, se sigue entonces que las premisas en forma de funciones de verdad de un argumento formal se afirman como si fuesen verdaderas en un sentido que no puede confirmarse sobre bases formales - lo cual seguramente es lo que deberíamos esperar.

(2) Sin embargo, el criterio unilineal proporciona una base para juzgar la falsedad. Porque si la relación de que se afirma que vale para un par $(p, q)$, excluye los valores de verdad de ese par $(p, q)$, éste es suficiente para probar que la relación no vale. Por otro lado, si son incluidos por la relación los valores de verdad de $(p, q)$, esto significa al menos que no se ha refutado que la relación valga para $(p, q)$. De este modo, en una línea deductiva de razonamiento formal, cuyas premisas se afirman como verdaderas, el criterio unilineal puede desempeñar un papel perfectamente válido como la base para inferir la continuación de la validez de una reclamación de verdad, por así decirlo.

Dada esta hipótesis muy general, se puede comenzar a vislumbrar una dirección para un análisis crítico cuya meta sea desarrollar y refinar esta hipótesis. Parece evidente que la reinterpretación del criterio unilineal tendrá que repercutir en la concepción de una tautología formal, como se entiende ésta ahora en lógica simbólica. En este problemático contexto se hará urgente la necesidad de un criterio lógico preciso para una regla de inferencia aceptable. Además, esto es para bien porque el problema de la validez de la Regla de Adición ya no puede evitarse. ${ }^{14}$ Esta regla es claramente incompatible con (1), arriba, porque permite en cualquier etapa de un argumento, la introducción de nuevas premisas con forma de funciones de verdad, sin otro apoyo que el del criterio unilineal. Así, el problema es: ¿existe algún criterio lógico para una regla de inferencia implicativa aceptable por medio de la cual pueda rechazarse la Regla de Adición? Este problema indudablemente no plantea ninguna dificultad insuperable. La principal dificultad ha sido justificar el planteamiento del problema dentro de la lógica simbólica. Una vez hecho esto, la inquietud lógica respecto a la Regla de Adición puede adquirir la vigencia que le corresponde.

Supóngase, por ejemplo, que pudieran aducirse bases lógicas rigurosas para el requisito de que una regla de inferencia, en su forma más simple, debe postular que algún par $(p, q)$, para el que ella vale, está relacionado contingentemente. Esta sugerencia puede a primera vista parecer arbitraria y excluir demasiado, pero considerémosla por mor del argumento. La Regla

14 La Regla de Adición puede expresarse así: de $Q$ infiérase ( $P \vee Q)$. Su forma implicativa es: $Q \supset(\mathbf{P}$ v $\mathbf{Q})$. Principia Mathematica postula esta regla como una proposición primitiva del cálculo proposicional elemental. Algunos lógicos posteriores han preferido considerarla como una "regla derivada" del sistema logico. 
de Adición -claro está- no satisface este requisito, porque de la premisa $Q$ no puede deducirse que $P$ y $Q$ no son lógicamente independientes la una de la otra. Mi argumento es, entonces, que, sea o no demasiado estrecho este requisito sugerido, sirve como ejemplo para mostrar la posibilidad de especificación de las propiedades formales de una tautología formal que puede hacer a esta última aceptable como regla de inferencia. En otras palabras, las tautologías formales difieren respecto a algunas de sus propiedades lógicas formales; y esto es relevante con respecto a si alguna tautología dada es o no una regla de inferencia aceptable.

Otro problema importante que no puede ignorarse es que, asł como está, el punto (1), arriba, no proporciona una explicación viable de todos los usos aceptables de las formas de funciones de verdad. Por ejemplo, (1) parece ser incompatible con el uso intuitivamente válido de la forma conjuntiva para unir cualesquiera proposiciones verdaderas, sin importar que alguna otra función de verdad valga o no también para estas proposiciones. Puesto que este uso de la forma conjuntiva desempeña un papel indispensable en la teoría misma de la deducción, si no puede justificarse de una manera consistente con (1), entonces la hipótesis expresada por medio de (1) y (2) carece de valor. Otro aspecto del problema general respecto a los usos de las formas de las funciones de verdad es que en muchos casos nos parece tener una elección en cuanto a en qué forma poner un par $(p, q)$. Por ejemplo, con frecuencia usamos la forma de la disyunción inclusiva para relacionar proposiciones que se encuentran entre sí en la relación de disyunción exclusiva. ¿Cómo ha de justificarse esta práctica de una manera que sea teóricamente consistente con (1)? O: ¿cómo podemos liberalizar (1), por así decirlo, sin caer de nuevo en la interpretación material?

Me parece que el problema aquí es que resulta inútil tratar de considerar el sentido en que un conjunto de condiciones de verdad vale para un par de proposiciones haciendo abstracción de la relación que se da entre las funciones proposicionales de que dichas proposiciones son instanciaciones. Cualquier intento de evitar esta consideración llevará a un callejón sin salida. Necesitamos una concepción completamente general de las relaciones-funciones de verdad entre funciones proposicionales elementales a fin de proporcionar una base teórica para el uso de las formas de las funciones de verdad. En relación con esto, deseo simplemente mencionar aquí un trabajo, con este enfoque, al que me he dedicado y que ahora se encuentra en una etapa avanzada.

Mi hipótesis inicial era que el conjunto de las dieciséis tablas de verdad básicas de Wittgenstein podría reinterpretarse como expresando las relaciones entre funciones proposicionales elementales, y que la relación que se da entre las instanciaciones pareadas de las funciones podría entonces ser derivable de un modo riguroso. Este enfoque de la cuestión me condujo enton- 
ces a algunas nuevas consideraciones. Por un lado, pronto se hizo claro que la misma forma de función de verdad podría ser el vehículo de más de una relación de función de verdad, como en el caso de la forma de tautología que es al mismo tiempo la forma de independencia lógica. Surgió entonces la cuestión de si la forma conyuntiva desempeña o no también tal papel dual. Por otro lado, este enfoque hace posible considerar el conjunto de relacionesfunciones de verdad que pueden valer para un par dado de proposiciones de una manera estrictamente concebida, sin apelar para nada al criterio unilineal.

El espacio no nos permite discutir más aquí cuestiones relacionadas con la reinterpretación de las tablas de verdad. He mencionado la posibilidad de esta perspectiva teórica sólo para indicar que el argumento de esta segunda parte del presente artículo puede no tener un resultado negativo. Este argumento puede resumirse como sigue: encontramos que la propia interpretación material de las funciones de verdad mismas contiene una ambigüedad fundamental que puede quizás resolverse haciendo explícito el pleno sentido lógico de las funciones de verdad y reinterpretando el papel lógico del criterio unilineal. Sobre esta base se formuló la hipótesis contenida en los puntos (1) y (2). Sin embargo, se hizo inmediatamente claro que esta hipótesis en turno requiere que reenfoquemos cuestiones teóricas, tan fundamentales como el criterio para una regla formal de inferencia aceptable y el sentido en que una relación-función de verdad puede valer para un par de proposiciones. De esta manera, llegamos a ver la necesidad de una construcción teórica para elucidar el concepto de las relaciones-funciones de verdad. Por otro lado, hemos encontrado una manera de reafirmar la intuitiva exigencia lógica de que una regla de inferencia debe cumplir el criterio de ser autoevidentemente válida.

La intención del presente artículo ha sido enfocar dos de los problemas más serios de la lógica formal contemporánea en el contexto de la hipótesis de que la lógica formal tiene una parte a priori consistente en nuestras certezas lógicas intuitivas, y una parte teórica cuyo principal propósito es proporcionar un sistema de lógica dentro del cual puedan operar nuestras certezas lógicas con creciente generalidad respecto a su aplicación. Aunque el presente artículo no se ha ocupado directamente de la aplicación de la lógica simbólica, el objetivo subyacente de la segunda parte ha sido destacar precisamente ese problema teórico que es el principal obstáculo para el uso de la lógica simbólica en el razonamiento sobre cuestiones empíricas. Porque hasta que se clarifique teóricamente en qué sentido una cierta relación-función de verdad, con preferencia a otra, vale para un par de proposiciones, la lógica simbólica no puede ser de gran utilidad para el científico que quiera poner en forma lógica una hipótesis empírica. Seguramente por esto la lógica sim- 
bólica sigue teniendo su principal aplicación en contextos analíticos, y aún no ha realizado en la práctica la plena generalidad de la idea central del cálculo proposicional elemental.

UNIVERSIDAd VeracruZANa

JUDITH SCHOENBERG

(trad. de Alejandro Herrera Ibáñez, revisada por la autora) 